\title{
INVESTIGATIONS ON THE RESISTANCE OF COMMENSAL SWINE ESCHERICHIA COLI TO SOME AMINOGLYCOSIDES-AMINOCYCLITOLS
}

\author{
Valentina Urumova ${ }^{1^{*}}$, Mihni Lyutskanov, Vladimir Petrov \\ Trakia University, Faculty of Veterinary Medicine, \\ Department of Microbiology, Infectious and Parasitic Diseases, \\ Stara Zagora, Bulgaria
}

\begin{abstract}
The aim of this study was to describe the prevalence of antibiotic resistance to some aminoglycosides, streptomycin, spectinomycin and gentamicin and three aminoglycoside- resistance genes in Escherichia coli isolated from feces and lagoon manure in six swine farms in Republic of Bulgaria. A total of 274 E. coli isolates from 270 fecal samples and twelve samples from lagoon manure were tested by disk diffusion method to determine resistance patterns to 11 antimicrobial agents. Aminoglycosides resistance also was determined by E-test, agar dilution method, PCR and qPCR. The highest resistance observed to streptomycin (70.0\%) and spectinomycin (65.5\%). Multi-resistance patterns in studied E. coli strains showed that the resistance to streptomycin/spectinomycin was most frequently seen together with resistance to ampicillin, tetracycline, and sulfonamides (39.6\%). The E. coli isolates resistant to streptomycin, spectinomycin were examined for the presence of $\operatorname{str} A / \operatorname{str} B$, aadA1 genes, and resistant isolates to gentamicin were evaluated for the presence of the aacC1 gene. The most common gene determining resistance to aminoglycosides was aadA 1 which was found in $54.0 \%$ of swine isolates and lagoon manure isolates followed by $\operatorname{straA} / \operatorname{str} B$ genes (32.3\%). The aacClgene was not identified in E. coli isolates resistant to gentamicin.
\end{abstract}

Keywords: aminoglycoside resistance, commensal Escherichia coli, pigs, lagoon manure

\footnotetext{
${ }^{1 *}$ E-mail: valentina_62@abv.bg
} 


\title{
ISPITIVANJE REZISTENCIJE KOMENSALNE ESCHERICHIA COLI KOD SVINJA NA NEKE AMINOGLIKOZIDE-AMINOCIKLITOLE
}

\author{
Valentina Urumova, Mihni Lyutskanov, Vladimir Petrov \\ Trakia University, Faculty of Veterinary Medicine, \\ Department of Microbiology, Infectious and Parasitic Diseases, \\ Stara Zagora, Bulgaria
}

\section{Kratki sadržaj}

Cilj ovog istraživanja je prikaz prevalencije rezistentnosti na antibiotike i to neke aminoglikozide, streptomicin, spektinomicin i gentamicin kao i tri gena Escherichia coli koji uslovljavaju rezistenciju na aminoglikozide , izolovanih iz fecesa i osoke iz sabirnih bazena na 6 farmi svinja u Republici Bugarskoj. Ispitano je 274 izolata E. coli dobijenih iz 270 uzoraka fecesa i 12 uzoraka osoke. Uzorci su testirani metodom disk difuzije u cilju odredjivanja modela rezistencije na 11 antimikrobnih agenasa. Rezistencija na aminoglikozide je takodje odredjivana primenom E-testa, metode dilucije agara, PCR i qPCR. Najveći stepen rezistencije ustanovljen je za streptomicin (70.0\%) i spektinomicin (65.5\%). Modeli višestruke rezistencije uočeni kod sojeva E. coli pokazali su da se rezistencija na streptomicin/spektinomicin najčešće javlja udružena sa rezistencijom na ampicilin, tetraciklin i sulfonamide (39.6\%). Izolati E. coli rezistentni na streptomicin i spektinomicin ispitani su na prisustvo gena $\operatorname{str} A / \operatorname{str} B, \operatorname{aad} A 1$, a izolati rezistentni na gentamicin na prisustvo gena $a a c C 1$. Ustanovljeno je da je rezistencija na aminoglikozide najčešće bila uslovljena genom aadA1 koji je detektovan kod 54.0\% izolata svinja i izolata osoke iz sabirnih bazena, a nakon njega slede geni straA/strB (32.3\%). Ben aacC1 nije identifikovan kod izolata $E$. coli rezistentnih na gentamicin.

Ključne reči: rezistencija na aminoglikozide, komensalna Escherichia coli, svinje, osoka iz sabirnih bazena

\section{INTRODUCTION}

The concept of EMA (2014) on the use of aminoglycosides in livestock and companion animals in the EU, development of resistance and public health risks is grounded on data about the increasing resistance to aminoglycosides in animal and human bacterial isolates. Data of EMA/ESVAC (2013) demonstra- 
te a widespread use of aminoglycosides in some animal species in particular (large and small ruminants, swine, horses, and pets) for treatment of septicaemic states, gastrointestinal infections, respiratory and urogenital infections. Some authors discussed the potential of resistant $E$. coli from domestic animals as a reservoir for the spread of resistance to aminoglycoside antibiotics among human population (Chaslus-Dancla et al., 1991; Jonson et al., 1994, 1995). The primary mechanism of aminoglycoside resistance is the production of aminoglycoside modifying enzymes. Three genetic determinants are associated to the expression of resistance to streptomycin in enterobacteria: ant $\left(3^{/ /}\right)-I a$ (synonym $\operatorname{aad} A$ ) coding for the production of adenyltransferase ANT (3/))-I, modifying streptomycin and spectinomycin, $a p h\left(3^{\prime \prime}\right)-I b$ (synonym strA), determining the production of the phosphoryltransferase APH $\left(3^{\prime /}\right)-\mathrm{I}$, modifying streptomycin and $a p h(6)-I d$ (synonym strB), responsible for the production of phosphoryltransferase APH (6)-I, that also modifies streptomycin (Heinzel et al., 1988; Hollingshead and Vapnek, 1985; Scholz et al., 1989). During the last years, reports about a new type of aminoglycoside resistance in animal bacterial isolates related to the prevalence of $16 \mathrm{~S}$ rRNA methylases and the respective high levels of resistance are increasing (Gonzalez-Zorn et al., 2005; Chen et al., 2007; Liu et al., 2008; Du et al., 2009; Davis et al., 2010; Hopkins et al., 2010; Deng et al., 2011). From modifying enzymes coding for resistance to gentamicin in E. coli strains from livestock, adenyltransferase ACC(3) - IV whose production is coded by the aac3-IV gene and that determines a combined resistance to gentamicin and apramycin, is of special interest. There are also data, although limited, on the prevalence of aacC1 and aac3-II genes in domestic animals (Guerra et al., 2003; Sãenz et al., 2004). These genes determine the production of acetyltransferases ACC (3)- I and AAC (3)-II, distinguished with their phenotype profile, which for the latter gene includes also resistance to tobramycin apart to gentamicin (Vaculenko and Mobashery, 2003).

\section{MATERIAL AND METHODS}

\section{Sample collection}

Between January 2013 and September 2014, 282 faecal swab samples were collected from different age groups of pigs (suckling, weaned, finisher) and lagoon manure from 6 farrow-to-finish farms. Faecal swabs were transported in Stuart Transport Medium (BD, USA) at low temperature within 18-24 hours.

\section{Culturing and identification of E. coli isolates}

Swabs were cultured on McConkey agar (Emapol, Poland) at $37^{\circ} \mathrm{C}$ for 24 
hours. Lactose-positive colonies were subcultured onto TSI agar (BD, USA) and submitted to preliminary biochemical typing via citrate utilisation, methyl red, Vogues Proskauer and indole production tests. The identification of strains was performed with kits for non-fermenting and enteric bacteria (BD, USA) and the semi-automated identification Crystal BBL system.

\section{Determination of the sensitivity of E. coli isolates to antibiotics}

The sensitivity of $E$. coli isolates to 11 chemotherapeutics was evaluated by the disk diffusion method as per CLSI, using Muller-Hinton agar (Emapol, Poland) and antibiotic disks (Emapol, Poland), loaded as followed: ampicillin $(10 \mu \mathrm{g})$, amoxicillin/clavulanic acid $(20 / 10 \mu \mathrm{g})$, cephalotin $(30 \mu \mathrm{g})$, ceftazidime $(10 \mu \mathrm{g})$, cefotaxime $(30 \mu \mathrm{g})$, gentamicin $(10 \mu \mathrm{g})$, streptomycin $(10 \mu \mathrm{g})$, spectinomycin $(25 \mu \mathrm{g})$, tetracycline $(30 \mu \mathrm{g})$, ciprofloxacin $(5 \mu \mathrm{g})$, sulfamethoxazole $(25 \mu \mathrm{g})$. The reference strain Escherichia coli ATTC 25922 was used for the control of disk diffusion method and MIC method.

The streptomycin MIC was determined in the agar dilution test and Muller-Hinton agar (Emapol, Poland), by preparation of doubling dilutions of streptomycin (Sigma-Aldrich) within 0.01-256 $\mu \mathrm{g} / \mathrm{mL}$. MIC for gentamicin was defined by E test strips (AB Biodisk, Solna Sweden). MICs were interpreted according to epidemiological criteria (EUCAST, www.eucast.org).

Determination of resistance genes to aminoglycoside in commensal E. coli

DNA extraction: For DNA extraction, 24-hour cultures incubated at $37^{\circ} \mathrm{C}$, respectively 3-4 colonies on McConkey agar were suspended in $100 \mu \mathrm{l}$ sterile distilled water free of inhibitors for molecular diagnostics (Qiagen). The DNA extraction kit DNeasy Blood Tissue Kit (Qiagen) was used.

Detection of resistance genes: The presence of resistance genes to aminoglycoside antibiotics, aadA1 was detected by qPCR and $\operatorname{str} A /$ str $B$ by PCR. The primers sequences for $s t r A / s t r B$ were strA-F ATGGTGGACCCTAAAACTCT and strB-R CGTCTAGGATCGAGACAAAG (Kozak et al., 2009). PCR assays in $25 \mu \mathrm{l}$ final volume contained $12.5 \mu \mathrm{l} \mathrm{Taq}$ PCR Master mix (Qiagen) and $3 \mu \mathrm{l}$ DNA template. The PCR reaction for $s t r A / s t r B$ consisted of an initial activation step at $94^{\circ} \mathrm{C}$ for $10 \mathrm{~min}$, followed by 30 cycles of DNA denaturation at $94^{\circ} \mathrm{C}$ for $30 \mathrm{~s}$, primer annealing at $63^{\circ} \mathrm{C}$ for $1 \mathrm{~min}$, and primer extension at $72^{\circ} \mathrm{C}$ for $30 \mathrm{~s}$, and final extension for $10 \mathrm{~min}$ at $72^{\circ} \mathrm{C}$. All reactions were carried out in Eppendorf gradient thermal cycler. Ten $\mu \mathrm{L}$ aliquots of PCR products were analyzed by gel electrophoresis with 1.5\% agarose gel (Peqlab, Germany). Gels were stained with ethidium bromide at concentration $10 \mu \mathrm{g} / \mathrm{mL}$ and visualized by UV transillumination. A $100 \mathrm{bp}$ DNA ladder plus (Qiagen) was used as marker. As positive control strain E. coli 94.4 was used, provided by Mrs. J. Mazurec from 
the Department of Molecular Biology, Faculty of Biological Sciences, University of Zielona Góra, Poland. Negative controls were PCR mixtures with the addition of water in place of template DNA.

To determine the aadAlgene, Microbial DNA qPCR Assay, aadA1 (Qiagen) was used. qPCR amplification was done with Stratagene Mx3000P instrument. The thermocycler protocol consisted of: initial PCR activation 1 cycle of $10 \mathrm{~min}$ at $95^{\circ} \mathrm{C}$, and 40 cycles of 2-step cycling - denaturation - 15 $\sec 95^{\circ} \mathrm{C}$, annealing and extension $2 \mathrm{~min} 60^{\circ} \mathrm{C}$. The results were interpreted according to manufacturer's instructions (negative control signal at CT $>35$ and $\mathrm{CT}=22 \pm 2$ for positive control).

\section{RESULTS}

Number of isolates: The total number of $E$. coli isolates from examined faecal swabs obtained from the different age categories and from lagoon manure at studied farms were 274 .

\section{Prevalence of antibiotic resistance by disk-diffusion method}

Tables 1, 2 and 3 present results from the phenotype analysis of resistance of $E$. coli isolates from the 6 surveyed farms to 11 chemotherapeutics. With respect to aminoglycosides, the highest resistance percentages to streptomycin and spectinomycin (93.2\% and $91.0 \%$ respectively) were observed in finisher pigs. Higher resistance to gentamicin (15.7\%) was established in E. coli isolates from weaned pigs than in finisher (7.9\%) or neonatal pigs (4.5\%).

Table1. Prevalence of antibiotic resistance in E. coli strains from suckling pigs from 6 farrow-to finish farms

\begin{tabular}{|c|c|c|c|c|c|c|c|}
\hline \multirow{2}{*}{ Antibiotic } & \multicolumn{7}{|c|}{ Resistant isolates \% } \\
\cline { 2 - 8 } & $\begin{array}{c}\text { Farm I } \\
\mathrm{n}=15\end{array}$ & $\begin{array}{c}\text { Farm II } \\
\mathrm{n}=14\end{array}$ & $\begin{array}{c}\text { Farm III } \\
\mathrm{n}=15\end{array}$ & $\begin{array}{c}\text { Farm IV } \\
\mathrm{n}=15\end{array}$ & $\begin{array}{c}\text { Farm V } \\
\mathrm{n}=15\end{array}$ & $\begin{array}{c}\text { Farm VI } \\
\mathrm{n}=15\end{array}$ & $\begin{array}{c}\text { Total } \\
\mathrm{n}=89\end{array}$ \\
\hline Ampicillin & $\begin{array}{c}1 \\
(6.7)\end{array}$ & $\begin{array}{c}3 \\
(21.4)\end{array}$ & $\begin{array}{c}6 \\
(40.0)^{*}\end{array}$ & $\begin{array}{c}3 \\
(20.0)\end{array}$ & $\begin{array}{c}2 \\
(13.3)\end{array}$ & $\begin{array}{c}2 \\
(13.3)\end{array}$ & $\begin{array}{c}17 \\
(19.0 .)\end{array}$ \\
\hline $\begin{array}{c}\text { Amoxi- } \\
\text { cillin/ cla- } \\
\text { vulanic acid }\end{array}$ & 0 & 0 & 0 & 0 & 0 & 0 & 0 \\
\hline Cephalotin & 0 & $\begin{array}{c}1 \\
(7.1)\end{array}$ & $\begin{array}{c}3 \\
(20.0)\end{array}$ & 0 & 0 & 0 & $\begin{array}{c}4 \\
(4.5)\end{array}$ \\
\hline
\end{tabular}




\begin{tabular}{|c|c|c|c|c|c|c|c|}
\hline \multirow{2}{*}{ Antibiotic } & \multicolumn{7}{|c|}{ Resistant isolates \% } \\
\cline { 2 - 8 } & $\begin{array}{c}\text { Farm I } \\
\mathrm{n}=15\end{array}$ & $\begin{array}{c}\text { Farm II } \\
\mathrm{n}=14\end{array}$ & $\begin{array}{c}\text { Farm III } \\
\mathrm{n}=15\end{array}$ & $\begin{array}{c}\text { Farm IV } \\
\mathrm{n}=15\end{array}$ & $\begin{array}{c}\text { Farm V } \\
\mathrm{n}=15\end{array}$ & $\begin{array}{c}\text { Farm VI } \\
\mathrm{n}=15\end{array}$ & $\begin{array}{c}\text { Total } \\
\mathrm{n}=89\end{array}$ \\
\hline Ceftazidime & 0 & 0 & 0 & 0 & 0 & 0 & 0 \\
\hline Cefotaxime & 0 & 0 & 0 & 0 & 0 & 0 & 0 \\
\hline Gentamicin & 0 & $\begin{array}{c}1 \\
(7.1)\end{array}$ & $\begin{array}{c}3 \\
(20.0)\end{array}$ & 0 & 0 & 0 & 4 \\
\hline $\begin{array}{c}\text { Strep- } \\
\text { tomycin }\end{array}$ & $\begin{array}{c}1 \\
(6.7)\end{array}$ & $\begin{array}{c}3 \\
(21.4)\end{array}$ & $\begin{array}{c}9 \\
(60.0)^{* *}\end{array}$ & $\begin{array}{c}3 \\
(20.0)\end{array}$ & $\begin{array}{c}4 \\
(26.6)\end{array}$ & $\begin{array}{c}2 \\
(13.3)\end{array}$ & $\begin{array}{c}22 \\
(24.7)\end{array}$ \\
\hline $\begin{array}{c}\text { Specti- } \\
\text { nomycin }\end{array}$ & 0 & $\begin{array}{c}2 \\
(14.3)\end{array}$ & $\begin{array}{c}7 \\
(46.6)^{*}\end{array}$ & $\begin{array}{c}2 \\
(13.3)\end{array}$ & $\begin{array}{c}4 \\
(26.6)\end{array}$ & 0 & $\begin{array}{c}15 \\
(16.8)\end{array}$ \\
\hline Tetracycline & $\begin{array}{c}3 \\
(20.0)\end{array}$ & $\begin{array}{c}9 \\
(64.3)^{* *}\end{array}$ & $\begin{array}{c}12 \\
(80.0)^{* * *}\end{array}$ & $\begin{array}{c}10 \\
(66.6)^{* *}\end{array}$ & $\begin{array}{c}5 \\
(33.3)\end{array}$ & $\begin{array}{c}8 \\
(53.3)^{*}\end{array}$ & $\begin{array}{c}47 \\
(52.8)\end{array}$ \\
\hline $\begin{array}{c}\text { Cipro- } \\
\text { floxacin }\end{array}$ & 0 & $\begin{array}{c}1 \\
(7.1)\end{array}$ & $\begin{array}{c}1 \\
(6.7)\end{array}$ & 0 & 0 & 0 & 2 \\
\hline $\begin{array}{c}\text { Sulfamet- } \\
\text { hoxazole }\end{array}$ & $\begin{array}{c}2 \\
(13.3)\end{array}$ & $\begin{array}{c}3 \\
(21.4)\end{array}$ & $\begin{array}{c}5 \\
(33.3)\end{array}$ & $\begin{array}{c}3 \\
(20.0)\end{array}$ & $\begin{array}{c}3 \\
(20.0)\end{array}$ & $\begin{array}{c}2 \\
(13.3)\end{array}$ & $\begin{array}{c}18 \\
(20.2)\end{array}$ \\
\hline
\end{tabular}

Legend: $\mathrm{p} \leq 0.05\left(^{*}\right) ; \mathrm{p} \leq 0.01\left({ }^{* *}\right) ; \mathrm{p} \leq 0.001\left(^{* *}\right)$

Data about the resistance of $E$. coli isolates to aminoglycosides in groups of suckling pigs showed the highest resistance to streptomycin, spectinomycin and gentamicin $(60 \%, 46.6 \%$, and $20 \%$ respectively) at farm 3 .

Isolates resistant to streptomycin and spectinomycin were the most prevalent (100\%) among weaned pigs from farms 3 and 5, followed by the occurrence of resistance to aminoglycosides in $86.6 \%$ of isolates from farms 4 and 6 and in $85.7 \%$ of isolates from farms 1 and 2. The percentage of isolates from weaned pigs resistant to gentamicin was the highest at farm $4(7.8 \%)$. 
Table 2. Prevalence of antibiotic resistance in E. coli strains from weaned pigs from 6 farrow-to finish farms

\begin{tabular}{|c|c|c|c|c|c|c|c|}
\hline \multirow{2}{*}{ Antibiotic } & \multicolumn{7}{|c|}{ Resistant isolates \% } \\
\hline & $\begin{array}{c}\text { Farm I } \\
\mathrm{n}=14\end{array}$ & $\begin{array}{c}\text { Farm II } \\
\mathrm{n}=15\end{array}$ & $\begin{array}{c}\text { Farm III } \\
\mathrm{n}=15\end{array}$ & $\begin{array}{c}\text { Farm IV } \\
n=15\end{array}$ & $\begin{array}{c}\text { Farm V } \\
n=15\end{array}$ & $\begin{array}{c}\text { Farm VI } \\
\mathrm{n}=15\end{array}$ & $\begin{array}{l}\text { Total } \\
\mathrm{n}=89\end{array}$ \\
\hline Ampicillin & $\begin{array}{c}3 \\
(21.4)\end{array}$ & $\begin{array}{c}10 \\
(66.6)^{\star *}\end{array}$ & $\begin{array}{c}15 \\
(100)^{\star * *}\end{array}$ & $\begin{array}{c}14 \\
(93.3)^{\star \star *}\end{array}$ & $\begin{array}{c}12 \\
(13.3)^{\star * *}\end{array}$ & $\begin{array}{c}6 \\
(40.0)\end{array}$ & $\begin{array}{c}60 \\
(67.4)\end{array}$ \\
\hline $\begin{array}{c}\text { Amoxi- } \\
\text { cillin/ cla- } \\
\text { vulanic acid }\end{array}$ & 0 & 0 & $\begin{array}{c}2 \\
(13.3)\end{array}$ & 0 & 0 & 0 & $\begin{array}{c}2 \\
(2.2)\end{array}$ \\
\hline Cephalotin & 0 & $\begin{array}{c}4 \\
(26.6)\end{array}$ & $\begin{array}{c}12 \\
(80.0)^{\star * \star}\end{array}$ & $\begin{array}{c}10 \\
(66.6)^{* * *}\end{array}$ & $\begin{array}{c}2 \\
(13.3)\end{array}$ & $\begin{array}{c}4 \\
(26.6)\end{array}$ & $\begin{array}{c}32 \\
(35.9)\end{array}$ \\
\hline Ceftazidime & 0 & 0 & 0 & 0 & 0 & 0 & 0 \\
\hline Cefotaxime & 0 & 0 & 0 & 0 & 0 & 0 & 0 \\
\hline Gentamicin & 0 & $\begin{array}{c}3 \\
(20.0)\end{array}$ & $\begin{array}{c}4 \\
(26.6) \\
\end{array}$ & $\begin{array}{c}7 \\
(46.6)\end{array}$ & 0 & 0 & $\begin{array}{c}14 \\
(15.7)\end{array}$ \\
\hline $\begin{array}{l}\text { Strep- } \\
\text { tomycin }\end{array}$ & $\begin{array}{c}12 \\
(85.7)\end{array}$ & $\begin{array}{c}12 \\
(85.7)\end{array}$ & $\begin{array}{c}15 \\
(100)^{\star}\end{array}$ & $\begin{array}{c}13 \\
(86.6)\end{array}$ & $\begin{array}{c}15 \\
(100)^{\star}\end{array}$ & $\begin{array}{c}13 \\
(86.6)\end{array}$ & $\begin{array}{c}80 \\
(89.8)\end{array}$ \\
\hline $\begin{array}{l}\text { Specti- } \\
\text { nomycin }\end{array}$ & $\begin{array}{c}12 \\
(85.7)\end{array}$ & $\begin{array}{c}12 \\
(85.7)\end{array}$ & $\begin{array}{c}15 \\
(100)^{\star}\end{array}$ & $\begin{array}{c}13 \\
(86.6)\end{array}$ & $\begin{array}{c}15 \\
(100)^{\star}\end{array}$ & $\begin{array}{c}13 \\
(86.6) \\
\end{array}$ & $\begin{array}{c}80 \\
(89.8)\end{array}$ \\
\hline Tetracycline & $\begin{array}{c}10 \\
(71.4)\end{array}$ & $\begin{array}{c}15 \\
(100)^{\star *}\end{array}$ & $\begin{array}{c}15 \\
(100)^{\star *}\end{array}$ & $\begin{array}{c}11 \\
(73.3)\end{array}$ & $\begin{array}{c}14 \\
(93.3)^{\star *}\end{array}$ & $\begin{array}{c}11 \\
(73.3)\end{array}$ & $\begin{array}{c}76 \\
(85.3)\end{array}$ \\
\hline $\begin{array}{l}\text { Cipro- } \\
\text { floxacin }\end{array}$ & 0 & $\begin{array}{c}2 \\
(13.3)\end{array}$ & $\begin{array}{c}3 \\
(20.0)\end{array}$ & 0 & $\begin{array}{c}1 \\
(6.7)\end{array}$ & 0 & $\begin{array}{c}6 \\
(6.7)\end{array}$ \\
\hline $\begin{array}{l}\text { Sulfamet- } \\
\text { hoxazole }\end{array}$ & $\begin{array}{c}5 \\
(35.7)\end{array}$ & $\begin{array}{c}14 \\
(93.3)^{\star * *}\end{array}$ & $\begin{array}{c}15 \\
(100)^{\star * *}\end{array}$ & $\begin{array}{c}11 \\
(73.3)^{\star}\end{array}$ & $\begin{array}{c}15 \\
(100)^{\star * *}\end{array}$ & $\begin{array}{c}12 \\
(80.0)^{\star *}\end{array}$ & $\begin{array}{c}72 \\
(80.8)\end{array}$ \\
\hline
\end{tabular}

Legend: $\mathrm{p} \leq 0.05\left(^{*}\right) ; \mathrm{p} \leq 0.01\left(^{* *}\right) ; \mathrm{p} \leq 0.001\left(^{* * *}\right)$

The highest resistance percentage to streptomycin and spectinomycin (100\%) was demonstrated in E. coli isolates from finisher pigs at farms 2, 3, 4 and 5 . With respect to gentamicin, the highest resistance $(28.5 \%)$ was found out among isolates from farm 3. 
Table 3. Prevalence of antibiotic resistance in E. coli strains from finishers pigs from 6 farrow-to finish farms

\begin{tabular}{|c|c|c|c|c|c|c|c|}
\hline \multirow{2}{*}{ Antibiotic } & \multicolumn{7}{|c|}{ Resistant isolates \% } \\
\hline & $\begin{array}{c}\text { Farm I } \\
\mathrm{n}=15\end{array}$ & $\begin{array}{c}\text { Farm II } \\
\mathrm{n}=15\end{array}$ & $\begin{array}{c}\text { Farm III } \\
\mathrm{n}=14\end{array}$ & $\begin{array}{c}\text { Farm IV } \\
\mathrm{n}=14\end{array}$ & $\begin{array}{c}\text { Farm V } \\
\mathrm{n}=15\end{array}$ & $\begin{array}{c}\text { Farm VI } \\
\mathrm{n}=15\end{array}$ & $\begin{array}{l}\text { Total } \\
\mathrm{n}=88\end{array}$ \\
\hline Ampicillin & $\begin{array}{c}1 \\
(6.7)\end{array}$ & $\begin{array}{c}3 \\
(20.0)\end{array}$ & $\begin{array}{c}4 \\
(28.5)\end{array}$ & $\begin{array}{c}3 \\
(21.4)\end{array}$ & $\begin{array}{c}3 \\
(20.0)\end{array}$ & $\begin{array}{c}4 \\
(26.6)\end{array}$ & $\begin{array}{c}18 \\
(20.3)\end{array}$ \\
\hline $\begin{array}{c}\text { Amoxi- } \\
\text { cillin/ cla- } \\
\text { vulanic acid }\end{array}$ & 0 & 0 & 0 & 0 & 0 & 0 & 0 \\
\hline Cephalotin & 0 & $\begin{array}{c}3 \\
(20.0)\end{array}$ & $\begin{array}{c}6 \\
(42.8)^{* *}\end{array}$ & $\begin{array}{c}2 \\
(14.2)\end{array}$ & $\begin{array}{c}3 \\
(20.0)\end{array}$ & $\begin{array}{c}1 \\
(6.7)\end{array}$ & $\begin{array}{c}15 \\
(17.0)\end{array}$ \\
\hline Ceftazidime & 0 & 0 & 0 & 0 & 0 & 0 & 0 \\
\hline Cefotaxime & 0 & 0 & 0 & 0 & 0 & 0 & 0 \\
\hline Gentamicin & 0 & $\begin{array}{c}2 \\
(13.3)\end{array}$ & $\begin{array}{c}4 \\
(28.5) \\
\end{array}$ & $\begin{array}{c}1 \\
(7.1)\end{array}$ & 0 & 0 & $\begin{array}{c}7 \\
(7.9) \\
\end{array}$ \\
\hline $\begin{array}{l}\text { Strep- } \\
\text { tomycin }\end{array}$ & $\begin{array}{c}10 \\
(66.6)\end{array}$ & $\begin{array}{c}15 \\
(100)^{\star * *}\end{array}$ & $\begin{array}{c}14 \\
(100)^{\star * *}\end{array}$ & $\begin{array}{c}14 \\
(100)^{\star * *}\end{array}$ & $\begin{array}{c}15 \\
(100)^{\star * *}\end{array}$ & $\begin{array}{c}14 \\
(93.3)^{\star}\end{array}$ & $\begin{array}{c}82 \\
(93.2)\end{array}$ \\
\hline $\begin{array}{l}\text { Specti- } \\
\text { nomycin }\end{array}$ & $\begin{array}{c}9 \\
(60.0)\end{array}$ & $\begin{array}{c}15 \\
(100)^{\star \star \star *}\end{array}$ & $\begin{array}{c}14 \\
(100)^{\star * \star}\end{array}$ & $\begin{array}{c}13 \\
(92.8)\end{array}$ & $\begin{array}{c}15 \\
(100)^{\star \star \star *}\end{array}$ & $\begin{array}{c}14 \\
(93.3)^{\star}\end{array}$ & $\begin{array}{c}80 \\
(91.0) \\
\end{array}$ \\
\hline Tetracycline & $\begin{array}{c}9 \\
(60.0)\end{array}$ & $\begin{array}{c}14 \\
(93.3)^{\star}\end{array}$ & $\begin{array}{c}14 \\
(100)^{\star * *}\end{array}$ & $\begin{array}{c}12 \\
(85.7)\end{array}$ & $\begin{array}{c}10 \\
(66.6)\end{array}$ & $\begin{array}{c}10 \\
(66.6)\end{array}$ & $\begin{array}{c}69 \\
(78.2)\end{array}$ \\
\hline $\begin{array}{l}\text { Cipro- } \\
\text { floxacin }\end{array}$ & 0 & $\begin{array}{c}1 \\
(6.7)\end{array}$ & $2(14.2)$ & 0 & $\begin{array}{c}1 \\
(6.7)\end{array}$ & 0 & $\begin{array}{c}4 \\
(4.5)\end{array}$ \\
\hline $\begin{array}{l}\text { Sulfamet- } \\
\text { hoxazole }\end{array}$ & $\begin{array}{c}4 \\
(26.6)\end{array}$ & $\begin{array}{c}8 \\
(53.3)\end{array}$ & $\begin{array}{c}13 \\
(92.8)^{\star * *}\end{array}$ & $\begin{array}{c}8 \\
(57.1)\end{array}$ & $\begin{array}{c}6 \\
(40.0)\end{array}$ & $\begin{array}{c}6 \\
(40.0)\end{array}$ & $\begin{array}{c}45 \\
(51.1)\end{array}$ \\
\hline
\end{tabular}

Legend: $\mathrm{p} \leq 0.05\left(^{*}\right) ; \mathrm{p} \leq 0.01\left(^{* *}\right) ; \mathrm{p} \leq 0.001\left(^{* * *}\right)$

Among multiresistant isolates, the highest prevalence of $23.6 \%$ was that of the phenotype profile including ampicillin, streptomycin, spectinomycin and tetracycline, following by the profiles of resistance to ampicillin, streptomycin, spectinomycin and sulfamethoxazole (9.7\%) and to ampicillin, cephalotin, gentamicin, streptomycin and tetracycline (6.3\%). 
Phenotypic analysis of MIC concentrations for streptomycin and gentamicin

Table 4 presents the cumulative MIC percentages to streptomycin and gentamicin. The MIC90 of isolates to streptomycin was $16 \mu \mathrm{g} / \mathrm{mL}$, whereas to gentamicin MIC90 was $1 \mu \mathrm{g} / \mathrm{mL}$.

Table 4. Distribution of MICs among commensal E. coli $(\mathrm{n}=274)$ isolated from pigs and lagoon manure

\begin{tabular}{|c|c|c|c|c|c|c|c|c|c|c|c|c|}
\hline Antibiotic & \multicolumn{12}{|c|}{ Cumulative (\%) MIC in $\mu \mathrm{g} / \mathrm{mL}$} \\
\hline & 0.06 & 0.125 & 0.250 & 0.5 & 1.0 & 2.0 & 4.0 & 8.0 & 16.0 & 32.0 & 64.0 & $128 \geq 256$ \\
\hline $\begin{array}{l}\text { Strep- } \\
\text { tomycin }\end{array}$ & & & 4.8 & 10.1 & 25.7 & 27.0 & 28.7 & 30.0 & 82.8 & 97.8 & 100 & \\
\hline Gentamicin & 58.3 & 82.7 & 85.3 & 88.3 & 98.5 & 98.9 & 98.9 & 100 & & & & \\
\hline
\end{tabular}

\section{Occurrence of resistance determinants}

Table 5 presents the prevalence of resistance genes $\operatorname{str} A / s \operatorname{tr} B$ and aadA1 among E. coli isolates from different categories of pigs and manure lagoons resistant to streptomycin and spectinomycin. The highest occurrence (54.0\%) was that of aadA 1 among isolates resistant to streptomycin and spectinomycin, whereas $32.3 \%$ of isolates were positive for $\operatorname{str} A / \operatorname{str} B$. The combination of aad $A 1$ and $s t r A / s t r B$ genes was determined in $3.1 \%$ of strains. The analysis of data on the distribution of resistance genes among the different age categories, the highest prevalence of aadA1 (23.4\%) was observed in finisher pigs, while $\operatorname{str} A / \operatorname{str} B$ genes were the most frequently encountered among weaned pigs. Isolates form suckling pigs also showed a higher prevalence of aadA1 (7.8\%). Higher prevalence of aadA1 (2.1\%) was established in E. coli isolates from manure lagoons as compared to $\operatorname{str} A / s t r B$ positive strains (1.0\%). The combination of aadA1 and $\operatorname{str} A / \operatorname{str} B$ was observed in $3.2 \%$ of resistant strains; similar were percentages $(1.5 \%, 1.0 \%)$ among isolates from weaned and finisher pigs. The same combination of resistance genes was not found out in isolates from suckling pigs.

None of E. coli strains resistant to gentamicin has exhibited the aacC1 gene. 
Table 5. Occurrence of resistance genes determined among commensal E. coli $(\mathrm{n}=$ 274) from pigs and lagoon manure

\begin{tabular}{|c|c|c|c|c|c|c|}
\hline \multicolumn{5}{|c|}{ Occurrence [n (\%)] } & \multirow[b]{2}{*}{ Total } & \multirow[b]{2}{*}{$95 \% \mathrm{CL}$} \\
\hline Genotype & $\begin{array}{l}\text { Suckling } \\
\text { pigs }\end{array}$ & $\begin{array}{l}\text { Weaned } \\
\text { pigs }\end{array}$ & Finishers & $\begin{array}{l}\text { Manure } \\
\text { lagoon }\end{array}$ & & \\
\hline $\begin{array}{c}\text { Resistance } \\
\text { to strep- } \\
\text { tomycin (\%) }\end{array}$ & $\begin{array}{c}22 \\
(24.7)^{\star * \star}\end{array}$ & $\begin{array}{c}80 \\
(89.8)^{\star \star *}\end{array}$ & $\begin{array}{c}82 \\
(93.2 .)^{* * *}\end{array}$ & $\begin{array}{c}8 \\
(2.9)\end{array}$ & $\begin{array}{l}192 \\
(70)\end{array}$ & $60.1 \div 79.0$ \\
\hline $\operatorname{aad} A 1$ & $\begin{array}{c}15 \\
(7.8) \\
\end{array}$ & $\begin{array}{c}40 \\
(20.8) \\
\end{array}$ & $\begin{array}{c}45 \\
(23.4) \\
\end{array}$ & $\begin{array}{c}4 \\
(2.1) \\
\end{array}$ & $\begin{array}{c}104 \\
(54.0)\end{array}$ & $46.9 \div 60.9$ \\
\hline $\operatorname{str} A / \operatorname{str} B$ & $\begin{array}{c}3 \\
(1.5)\end{array}$ & $\begin{array}{c}30 \\
(15.6)\end{array}$ & $\begin{array}{c}27 \\
(14.1)\end{array}$ & $\begin{array}{c}2 \\
(1.0)\end{array}$ & $\begin{array}{c}62 \\
(32.3)\end{array}$ & $25.9 \div 39.0$ \\
\hline $\begin{array}{c}\operatorname{aad} A 1+\operatorname{str} A / \\
\operatorname{str} B\end{array}$ & - & $\begin{array}{c}3 \\
(1.5)\end{array}$ & $\begin{array}{c}2 \\
(1.0)\end{array}$ & $\begin{array}{c}1 \\
(0.5)\end{array}$ & $\begin{array}{c}6 \\
(3.1)\end{array}$ & $1.1 \div 6.0$ \\
\hline
\end{tabular}

Legend: $\left.\mathrm{p} \leq 0.05\left(^{*}\right) ; \mathrm{p} \leq 0.01\left(^{* *}\right) ; \mathrm{p} \leq 0.001{ }^{* * *}\right)$

\section{DISCUSSION}

Molecular characteristics of the commonest co-resistant phenotypes in commensal $E$. coli isolates from domestic animals are related to the presence of bla TEM-1 coding resistance to ampicillin, aadA1 and strA/strB determining streptomycin resistance, tet $(A)$ and tet $(B)$ in tetracycline-resistant strains, sul1 in sulfamethoxazole-resistant and $d f r A 1$ - in trimethoprim-resistant isolates. The resistance to gentamicin among commensal poultry and swine E. coli isolates is outlined with an ascending trend (Szmolka et al., 2013). The authors presented data from the Hungarian Antimicrobial Monitoring System, showing that the resistance to gentamicin among commensal porcine E. coli isolates kept the usual low levels throughout the monitoring period from 2004 to 2008. In their view, the prevalence of $s t r A$, strB, and aadA1 genes among porcine $E$. coli strains was within the ranges $30 \%-60 \%, 60 \%-100 \%$, and $1-30 \%$ respectively. Sundin et al. (1996) commented the wide spread of $s t r A / s t r B$ genes in the environment and the relationship between their spread and exchange in the different ecological niches, plants, livestock animal species and humans. Sandvang et al. (2000) and Jakobsen et al. (2007) also discussed the incidence of ant (2/I) -I, aac (3)-IIa, and aac(3)-IVa among gentamicin-resistant E. coli 
strains from pigs and other domestic animals. Guerra et al. (2003) presented the prevalence of aadA1 (61\%) and strA/strB (59\%) resistance genes in commensal E. coli from poultry, swine and cattle. Mazurec et al. (2013) detected the presence of aadA1 in $35.0 \%$ of streptomycin-resistant commensal porcine E. coli isolates.

Data about the resistance to streptomycin among commensal E. coli isolates from pigs show a substantial variability. For instance Wasyl et al. (2007) reported the presence of resistance in $37.0 \%$ commensal porcine $E$. coli isolates while streptomycin resistance among isolates from weaned animals reported by Stannarius et al. (2009) was $60.6 \%$. The results of Mazurec et al. (2013) with respect to streptomycin resistance showed that it was present in $88.3 \%$ of tested commensal E. coli strains from swine.

The prevalence of resistance to streptomycin (70.0\%) and spectinomycin (65.5\%) among commensal porcine E. coli isolates in the present study was comparable to the results of Stannarius et al. (2009) and Mazurec et al. (2013). It should be noted that according to our data, the resistance to gentamicin (12.4\%) was considerably higher that reported by Szmolka et al. (2012).

The percentage of $\operatorname{str} A / s t r B$ positive streptomycin-resistant strains in this study (32.3\%) was the same as results presented by Szmolka and Nagy (2013), yet the occurrence of the aadA1 gene (54.0\%) was higher.

\section{CONCLUSION}

The established resistance to streptomycin and spectinomycin to commensal E. coli strains from pigs was close to the highest percentages reported by different research teams from the EU member states. As the genetic resistance profile was concerned, the prevalence of the aadA1 genes was incontestable among isolates from the different age categories of pigs and environmental strains (from manure lagoons). The lack of aacC1 genes from the genetic profile of resistance to gentamicin was not an exception as could be seen from limited data on their prevalence among domestic animals and at present, the data for the occurrence of these genes in commensal porcine E. coli strains are predominantly from the Asia region.

\section{LITERATURE}

1. Chaslus-Dancla E., Pohl P., Meurisse M., Marin M., Lafont J.P.: High genetic homology between plasmids of human and animal origins conferring resistance to the aminoglycosides gentamicin and apramycin. Antimicrob 
Agents Chemother, 35, 590-593, 1991.

2. Chen L., Chen Z.L., Liu J.H., Zeng Z.L., Ma J.Y., Jiang H.X.: Emergence of RmtB methylase-producing Escherichia coli and Enterobacter cloacae isolates from pigs in China. J Antimicrob Chemother, 59, 880-885, 2007.

3. Davis M.A., Baker K.N., Orfe L.H., Shah D.H., Besser T.E., Call D.R.: Discovery of a gene conferring multiple-aminoglycoside resistance in Escherichia coli. Antimicrob Agents Chemother, 54, 2666-2669, 2010.

4. Deng Y., He L., Chen S., Zheng Z., Zeng Z., Liu Y., Sun Y., Ma J., Chen Z., Liu J.H.: F33:A-B and F2; A-B; -plasmids mediate dissemination of $r m t B$ bla CTX-M-9 group genes and $r m t B$-qepA in Enterobacteriaceae isolates from pets in China. Antimicrob Agents Chemother, 55, 4926-4929, 2011.

5. Du X.D., Wu C.M., Liu H.B., Li X.S., Beier R.C., Xiao F., Qin S.S., Huang S.Y., Shen J.Z.: Plasmid-mediated ArmA and RmtB 16S rRNA methylases in Escherichia coli isolated from chickens. J Antimicrob Chemother, 64, 1328-1330, 2009.

6. EMA/ESVAC.: European Medicines Agency, European Surveillance of Veterinary Antimicrobial Consumption. Sales of antimicrobial agents in 25 EU/EEA countries in 2011. (EMA/236501/2013). Third ESVAC report. In http://www.ema.Europa.eu/dosc/en GB/document library/Report/2013/10/WC500152311.pdf.99., 2013.

7. Gonzalez-Zorn B., Teshager T., Casas M., Porrero M.C., Moreno M.A., Courvalin P., Dominguez L.: armA and aminoglycoside resistance in Escherichia coli. Emer Infect Dis, 11, 964-956, 2005.

8. Guerra B., Junker E., Schroeter A., Malorny B., Lehmann S., Helmuth R.: Phenotypic and genotypic characterization on antimicrobial resistance in German Escherichia coli isolates from cattle, swine and poultry. J Antimicrob Chemother, 52, 489-492, 2003.

9. Heinzel P., Werbitzky O., Distler J., Peipersberg W.: A second streptomycin resistance gene from Streptomyces griseus codes for streptomycin - $3^{\prime \prime}$ phosphotransferase. Arch Microbiol, 150, 184-192, 1988.

10. Hollingshead S., Vapnek D.: Nucleotide sequence analysis of the gene encoding a streptomycin/spectinomycin adenylyltransferase. Plasmid, 13, 17-30, 1985.

11. Hopkins K.L., Escudero J.A., Hidalgo L., Gonzalez-Zorn B.: 16S rRNA methyltransferase RmtC in Salmonella enterica serovar Virchow. Emer Infect Dis, 16, 712-715, 2010.

12. Jakobsen L., Sandvang D., Jensen V.F., Seyfarth A.M., Frimodt-Müller N., Hammerum A.M.: Gentamicin susceptibility in Escherichia coli related to the genetic background: problems with breakpoints. Clinical Microbiol Infect, 13, (8), 816-842, 2007. 
13. Johnson A.P., Burns L., Woodford N., Threlfall E.J., Naidoo J., Cooke E.M., George R.C.: Gentamicin resistance in clinical isolates of Escherichia coli encoded by genes of veterinary origin. J Med Microbiol, 40, 221-226, 1994.

14. Johnson A.P., Malde M., Woodford N., Cunney R.J., Smyth E.G.: Urinary isolates of apramycin-resistant Escherichia coli and Klebsiella pneumoniae from Dublin. Epidemiol Infect, 114, 105-112, 1995.

15. Kozak G.K., Boerlin P., Janecko N., Reid-Smith R.J., Jardine C.: Antimicrobial resistance in Escherichia coli isolates from swine and wild small mammals in the proximity of swine farms and in natural environments in Ontario. Appl Environ Microbiol, 76, (3), 559-566, 2009.

16. Liu J.H., Deng Y.T., Zeng Z.L., Gao J.H., Chen L., Arakawa Y., Chen Z.L.: Coprevalence of plasmid-mediated quinolone resistance determinants QepA, Qnr, and AAC(6')-Ib-cr among $16 \mathrm{~S}$ rRNA methylase RmtB-producing Escherichia coli isolates from pigs. Antimicrob Agents Chemother, 52, 2992-2993, 2008.

17. Mazurec J. Pusz P., Bok E., Stosik M., Baldy- Chudzik K.: The phenotypic and genotypic characteristics of antibiotic resistance in Escherichia coli populations isolated from farm animals with different exposure to antimicrobial agents. Polish J Microbiol, 62, (2), 173-179, 2013.

18. Sãenz Y., Brinas L., Domínguez E., Ruiz J., Zarazaga M., Vila J., Torres C.: Mechanisms of resistance in multiple-antibiotic resistant Escherichia coli strains of human, animal, and food origins. Antimicrob Agents Chemother, 48, (10), 3996-4001, 2004.

19. Sandvang D., Aarestrup F.M. : Characterization of aminoglycoside resistance genes and class 1 integrons in porcine and bovine gentamicin-resistant Escherichia coli. Microb Drug Resist, 6, (1), 19-27, 2000.

20. Sholz P., Haring V., Wittmann-Liebold B., Ashman K., Bagdasarian M., Scherzinger E.: Complete nucleotide sequence and gene organization of the broad-host range plasmid RSF1010. Gene, 75, 271-288, 1989.

21. Stannarius C., Bürgi E., Regula G., Zychowska M.A., Zweifel C., Stephan R.: Antimicrobial resistance in Escherichia coli strains isolated from Swiss weaned pigs and sows. Schweiz Arch Tierheilk, 151, 119-125, 2009.

22. Sundin G.W., Bender C.L.: Dissemination of the strA-strB streptomycinresistance genes among commensal and pathogenic bacteria from humans, animals, and plants. Molecul Ecology, 5, 133-143, 1996.

23. Szmolka A., Anjum M.F., La Regione R.M., Kaszanyitzky E.J., Nagy B.: Microarray based comparative genotyping of gentamicin resistant Escherichia coli strains from food animals and humans. Vet Microbiol, 156, 110$118,2012$. 
24. Szmolka A., Nagy B.: Multidrug resistant commensal Escherichia coli in animals and its impact for public health. Frontiers Microbiol, doi:10.3389/ fmicb. 2013.00258, 2013.

25. Vakulenko S., Mobashery S.: Versatility of aminoglycosides and prospects for their future. Clin Microbiol Rev, 16, 430-450, 2003.

26. Wasyl D., Hoszowski A.: Safepork, Session 6: Antimicrobial resistance, 506- 509, Verona, Italy, 2007.

Primljeno: 15.04.2015.

Odobreno: 02.06.2015. 